\title{
IMPUESTOS DIRECTOS: PROGRESIVIDAD OPTIMA
}

\author{
JESÚS SEADE* \\ El Colegio de México
}

\section{INTRODUCCIÓN: CONTROL DE LA DISTRIBUCIÓN}

UN POSTULADO BÁSICO EN ECONOMÍA del bienestar dice que, bajo supuestos usuales, todo estado Pareto-óptimo de la economía puede alcanzarse como un equilibrio competitivo descentralizado, siempre y cuando el ingreso fijo (propiedad) de los individuos pueda ser previamente redistribuido mediante impuestos fijos ("lump-sum taxes") ideales, desligados de toda decisión económica de los individuos. El problema es que, desafortunadamente, la aplicación de este teorema requiere una información mayor de la que autoridad hacendaria alguna tiene o puede esperar conseguir, a saber, las preferencias detalladas y el ingreso potencial de cada miembro de la sociedad.

El gobierno no puede obtener datos precisos del "ingreso total"1 (y habilidades específicas de distintos tipos, preferencias, necesidades) de cada individuo, sino sólo a través de indicadores económicos indirectos que están bajo el control del individuo mismo, tal como su ingreso (que depende de la elección del tipo e intensidad de trabajo que éste hace, asunción de riesgos, etc.). Como resultado, ninguna redistribución no distorsionadora es normalmente posible: el statu quo, cualquiera que éste sea, en ausencia de intervención fiscal, es el único punto Pareto-óptimo interesante ${ }^{2}$ y accesible.

Un economista ultra liberal concluiría, que la intervención estatal debe reducirse a cero (o en forma más realista, al mínimo posible compatible con otras necesidades o consideraciones), so pena de colocar a la economía en un punto ineficiente, en el sentido de Pareto. Pero ésta es una posición equivocada, o correcta pero basada en la adopción implícita de un conjunto de valores extremo, especial: aquél en el que la distribución no importa y un peso es un peso donde

* Partes de este artículo están basadas en Seade (1977) (consultar Referencias), trabajo en el cual recibí comentarios muy útiles de A. B. Atkinson, J. S. Flemming, J. A. Mirrlees y N. H. Stern.

1 Máximo ingreso potencial por trabajo, capitalizado, más herencias y otras transferencias.

2 Es decir, excluyendo soluciones Pareto-eficientes alternativas que pueden obtenerse mediante la fijación de impuestos fijos arbitrarios desligados de los objetivos del gobierno, p. ej. determinados al azar. 
quiera que éste se halle. Sin duda el extremo opuesto -aquél en que la igualdad reina cualquiera que sea su costo-manifiesta su escaso interés: es fácil dar ejemplos en donde esto sólo se puede lograr con consumo equitativamente nulo para todos.

Múltiples opciones se abren al gobierno: una de ellas consiste en disminuir el tamaño del pastel social a cambio de lograr una mejor distribución del mismo. El problema es encontrar el balance correcto entre los objetivos "eficiencia" y "distribución". Este es el tema central de la teoria de imposición óptima, que busca modelar en una forma práctica, estudiable, el efecto que determinados impuestos $u$ otros instrumentos de control tienen sobre los incentivos individuales, sobre la eficiencia, para así determinar endógenamente la frontera de Pareto alcanzable (con los instrumentos dados) y de ahí escoger el mejor estado de la economía, en donde "mejor" se juzga en relación al conjunto de valores del individuo o institución que elabora políticas, plasmados en una función de bienestar social. $^{3}$

La teoría de impuestos óptimos (con tasa variable) se inicia con Mirrlees (1971), que mediante un modelo simple, especial (sólo un bien de consumo; consumidores con preferencias idénticas y sólo diferentes en sus salarios; trabajo en esencia homogéneo), se aboca por primera vez en forma rigurosa a la vieja discusión ${ }^{4}$ sobre los efectos (recaudación, distribución, eficiencia) del impuesto sobre el ingreso. En este artículo ex tendemos el modelo a realidades económicas (supuestos) y a interpretaciones (presencia de otros impuestos directos y/o de precios no uniformes de electricidad, alimentos, etc.) más generales, y sobre todo presentamos y discutimos un resultado general para estos modelos [demostrado en Seade (1977)] que tiene importantes consecuencias para el diseño de impuestos y para nuestra comprensión intuitiva del problema. El modelo es aún un tanto especial pero ilustrativo, y no está basado en supuesto extraño alguno del cual dependa su comportamiento. Nuestro propósito principal es extraer, del modelo formal especial, observaciones con contenido económico intuitivo de más amplia aplicabilidad referentes al diseño "óptimo" o "eficiente" de impuestos directos, en busca de argumentos de interés para el diseño de estos impuestos más que de recetas o descripciones detalladas de los mismos.

\section{EL MODELO}

Consideremos una economía cuyos miembros difieren en un solo parámetro h, que puede interpretarse como riqueza (fija), algún índice de "habilidad de

3 Para facilitar la exposición estamos implícitamente suponiendo, en este párrafo, que el gobierno es paretiano. Este supuesto, con el que trabajamos en este artículo, es usual en imposición óptima y común en economía del bienestar más en general, pero para ciertos usos o aspectos es restrictivo, como arguye Sen (1973). El caso más general, que presenta características propias interesantes, se estudia en Seade (1980), en el cual se da atención especial a la aplicación a la maximización de ganancias bajo precios no uniformes. La extensión correspondiente puede verse en Seade (1979) en preparación en español.

4 Véase, p. ej., Pigou (1929): págs. 87-97; 713-719. 
mercado" (ingreso potencial), patrones de comportamiento (preferencias), o cualquier diferencia central entre consumidores en la cual podamos querer concentrarnos en aplicaciones específicas del modelo. $\mathrm{Si}$ algunos de estos factores han de variar simultáneamente (habilidad y propensión al trabajo, por ejemplo), lo hacen en forma perfectamente correlacionada, de modo que un solo parámetro basta.

Puede demostrarse que, en términos generales, si h toma un número finito de valores el impuesto óptimo será "hecho a la medida" para la población específica que se tiene, enfrentando a cada consumidor con una esquina (aumento súbito del impuesto marginal en el nivel dado) de la curva de impuestos. Por otro lado, aunque toda economía es de hecho finita, el gobierno no tiene información precisa de los valores de $\mathrm{h}$ que se dan, sino sólo información estadística de la forma en que esta variable está distribuida. Por ambas razones, es natural y conveniente tratar a $h$ como una variable continua, distribuida con densidad $f(h)$ en el intervalo $[\underline{h}, \bar{h}]$, donde $\underline{h}$ y $\bar{h}$ son los extremos de $h$ presentes $(f>0)$ en la población.

Puesto que $h$ representa toda forma en que los consumidores difieren entre sí, éstos deben tener las mismas preferencias, excepto por lo que a h se refiere, sobre los $\mathrm{N}$ bienes de consumo el vector de cuyas cantidades denotamos por $\underline{\mathrm{x}}$. Si Tas preferencias son continuas y varían en forma continua con $h$, serán representa. bles por una función de utilidad $u(x ; h)$. Finalmente, en lo que concierne a la descripción de los consumidores, adopto la convención de que, a diferencia de sus preferencias, todos los consumidores tienen restricciones presupuestarias idénticas. ${ }^{5}$ Esto simplemente requiere, primero, que definamos los bienes $\underline{x}$ en la forma en que éstos aparecen en la producción (número de unidades de trabajo ajustadas por productividad, y no simples horas trabajadas, por ejemplo) y segundo, que midamos $\mathrm{x}$ como transacciones netas con el mercado, y no como consumo total. Así, si las dotaciones iniciales de bienes (o ingreso) de hecho varían con $h$, estas diferencias simplemente afectan la utilidad obtenida de las transacciones $\underline{x}$ una vez adicionadas a (o substraídas de) las dotaciones iniciales, si el consumo final es lo que importa. Esto ya está contemplado al escribir $u(\underline{\mathrm{x}} ; h)$.

Es conveniente ver, a través de un par de ejemplos, la forma en que esta "transferencia de diferencias", de presupuestos a preferencias, opera.

Primero, para estudiar el impuesto al ingreso, es natural ignorar la diversidad de bienes de consumo y concentrarse en las selecciones de pares consumo agregado-trabajo de los individuos: $U(c, l)$, o quizás $U(c, l, h)$, donde $c$ es consumo y $l$, digamos, horas trabajadas. $\mathrm{Si} h$ representa el salario por hora, ingreso es $h l$ y la restricción presupuestaria sobre el consumo, dada una función de impuestos $I(h l)$, está dada por $c=h l-I(h l)$, que depende de $h$. Sin embargo, introduciendo la variable "ingrtso" representada por y $(=h l)$, y redefiniendo preferencias en términos de ésta y no de "horas" (o esfuerzo), $U(c, l)$ se convierte en $U(c, y / h)$, que es la forma especial que nuestra $u(c, y ; h)$ toma en este ejemplo. En tanto, la restricción presupuestaria pasa a ser la misma para toda $h: c=y-I(y)$. Este modelo

5 No ingresos idénticos, sino por ejemplo idéntica dependencia del ingreso neto sobre el bruto, es decir mismo conjunto de combinaciones $x$ permisibles, de entre las cuales se ha de escoger la $x$ propia. 
(más trabajando en términos de $c, l$ ), es el utilizado por Mirrlees (1971) en su estudio original del impuesto al ingreso.

Otro ejemplo específico de interés es aplicable a la política de precios de una empresa (pública o privada, excepto que en estas notas nos referimos sólo al objetivo utilitarista, que difícilmente puede ser privado). En este caso es conveniente ignorar decisiones de trabajo, tomando el ingreso personal como fijo, dado directamente por $h$, y es necesario desagregar $c$ (consumo) un poco, para singularizar el consumo $b$ del producto de la empresa en cuestión. Si $c$ representa ahora consumo "de otras cosas" (medido en unidades tales que su precio es 1$)$ y $P(b)$ el precio total a pagar por la cantidad $b$, la restricción presupuestaria es $c+\mathrm{P}(b)=h$, que desde luego varía con $h$, mientras que las preferencias están dadas por $U(c, b)$ [o $U(c, b, h)$ ]. Sin embargo, introduciendo la variable $a$ para aquello que el consumidor da a la empresa "a cambio" de $b$, o sea $a \equiv P(b)$, podemos transfor$\operatorname{mar} c+P(b)=h$ en $c=h-P(b)=h-a$. Sustituyendo en $U(c, b)$, ésta se transforma en $U(h-a, b)$, que es la forma especial que $u(a, b ; h)$ toma en este caso. La restricción, entre tanto, se convierte en $a=P(b)$, igual para toda $h$.

Pasemos ahora a la descripción del comportamiento de los consumidores. El gobierno controla, a través de impuestos, el conjunto de puntos $\underline{x}$ de entre los cuales los consumidores pueden realizar su selección; llamemos a este conjunto $\theta$. El problema para cada persona $\mathrm{h}$ es, por tanto, el de escoger la $\underline{x}$ que prefiere en $\theta$ :

$$
\underset{\mathrm{x}}{\operatorname{maximizar}} u(\mathrm{x} ; h) \text { sujeto a } \underline{\mathrm{x}} \epsilon \theta .
$$

Este problema, escrito así, no admite solución fácil. Sin embargo, esta optimalidad del $\underline{x}$ escogido por un determinado $h$ sobre todos los otros puntos en $\theta$ exige que, en particular, ese punto sea preferido sobre los puntos escogidos por otros consumidores. Es decir, escribiendo $\underline{x}(h)$ para la selección de una persona $h,(1)$ requiere que $\underline{x}(h)$ resuelva

$$
\max _{h^{\prime}} u\left(\mathrm{x}\left(h^{\prime}\right) ; h\right)
$$

que de hecho dice que $h$ puede encontrar su óptimo decidiendo "como quién" debería actuar, o sea decidiendo el vector $\mathrm{x}$ de cual $h^{\prime}$ (corriendo $h^{\prime}$ a lo largo de $[h, h]$ ) tomar. Esta expresión nos dice que, en el $h^{\prime}$ optimo para $h$ (que por definición debe ser en $h^{\prime}=h$ misma),

$$
u_{\underline{\mathrm{x}}} \cdot \underline{\mathrm{x}}^{\prime}=0
$$

que se obtiene derivando (2) con respecto a $h^{\prime}$ e igualando a cero, y en la cual $u_{\mathrm{x}}$ representa el vector hilera de utilidades marginales $\mathrm{y} \underline{\mathrm{x}}$ ' el vector columna de derrivadas de la función de consumos $\underline{x}(h)$. Lo que esta condición exige es que para cualquier cambio pequeño en los consumos óptimos $x$ (h) (en direcciones 
tomadas por individuos con mayor o menor $h$ ), $d u=0$, y por tanto que la curva o superficie de indiferencia del individuo en cuestión sea tangencial, en ese punto, a la superficie de $\theta$. Esto se ve más claro en el caso $N=2$, en el cual (3) se reduce a

$$
u_{x_{1}} x_{1}^{\prime}+u_{x_{2}} x_{2}^{\prime}=0
$$

la cual se puede reescribir como

$$
-\frac{u_{x_{1}}}{u_{x_{2}}}=\frac{d x_{2} / d h}{d x_{1} / d h}=\frac{d x_{2}}{d x_{1}}
$$

sobre $\theta$.

Habiendo completado la descripción de los consumidores y de su comportamiento, ${ }^{6}$ podemos pasar brevemente a los otros componentes del modelo.

El gobierno o empresa pública es, suponemos, utilitarista, es decir, está interesado en la maximización de

$$
W=\int_{\underline{\mathrm{h}}}^{\bar{h}} u[\underline{\mathrm{x}}(h), h] f(h) d h .
$$

Hay una complicación que no necesita ser introducida explícitamente: que, en principio, el gobierno puede querer utilizar un índice de preferencias individuales diferente Paretiano pues estamos suponiendo que lo somos pero diferente de la cardinalización $u$ dada, digamos $G(u)\left(G^{\prime}>O\right)$, que puede incluso no ser el mismo para diferentes valores de $h$, o sea $G(u, h)$ más en general (con $G_{u}>0$ ). Así, una versión "muy cóncava" de $G$ representará una posición fuertemente redistributiva (mayor peso a individuos con baja utilidad). Pero esto sería una complicación inútil de nuestra notación, pues podemos tomar u misma en (4) como el índice $G(u, h)$ específico adoptado por el gobierno de acuerdo a sus preferencias.

Técnicamente, el problema es llevar a cabo una selección óptima, según la evaluación (4), de $N$ funciones x $(h)$ que satisfagan la condición para descentralización (3) (asociada a las condiciones de segundo orden individuales a que ya nos hemos referido, que normalmente serán satisfechas con holgura y podemos ignorar) así como una restricción a las demandas agregadas, que puede ser interpretada como una restricción al producto total (ne to de demandas gubernamentales), a la recaudación requerida por el erario, o a las ganancias mínimas (subsidio requerido máximo) permisibles a una empresa pública dada ${ }^{7}$ :

6 Estamos ignorando condiciones de segundo orden y otros puntos de rigor que no afectan lo que se ha dicho ni agregan mayor cosa de especial interés.

7 La primera interpretación de (5) es obvia: una función de producción linealizada. Para la segunda, si escribimos el impuesto total que un individuo $h$ paga como $I(h)$ y su ingreso fijo (aparte de ingreso por trabajo posible) como $F$, tenemos que su restricción presupuestaria se puede escribir como $p \cdot x+I=F$ la cual, imponiendo $\int I \geqslant R$ (requerimiento del erario), nos da (5), con $A \equiv \int F-$ R. Finalmente, para leer (5) como "ganancias $\geqslant-A$ ", tomamos 


$$
\int_{\underline{\mathrm{h}}}^{\bar{h}} \underline{\mathrm{p}} \cdot \underline{\mathrm{x}}(h) f(h) d h \leq A,
$$

donde $\underline{p}$ es el vector de precios sombra relativos $\left(\operatorname{con} p_{1}=1\right)$ en el equilibrio óptimo.

\section{IMPUESTOS ÓPTIMOS: CARACTERIZACIÓN}

Para maximizar (4) sujeto a (3) y (5), escribimos el Lagrangeano:

$$
L \equiv \int_{\underline{\mathrm{h}}}^{\bar{h}}\left\{[u(\underline{\mathrm{x}}, h)-\lambda \underline{\mathrm{p}} \cdot \underline{\mathrm{x}}] f+\mu u_{\underline{\mathrm{x}}} \cdot \underline{\mathrm{x}}{ }^{\prime}\right\} d h,
$$

donde $\mu \equiv \mu(h)$ es un multiplicador-función, pues (3) debe ser satisfecha para cada $h$. La solución del problema, de cálculo de variaciones, presenta poco interés en sí misma y será omitida [v. Seade (1977)].

Las condiciones de primer orden y condiciones de transversalidad (para los puntos extremos) son (3), (5), además de

$$
\left(u_{\underline{\mathrm{x}}}-\underline{\mathrm{p}}\right) f=\mu^{\prime} u_{\underline{\mathrm{x}}}+\mu u_{\underline{\mathrm{x}}} h
$$

(donde $u_{\underline{x} h}$ es el vector de $\partial^{2}$

$$
\mu(\underline{h})=\mu(\bar{h})=0 .
$$

La ecuación (6) tiene una dependencia indeseable del nivel de precios: si duplicamos toda $u$ y por tanto los precios sombra, el lado izquierdo de (6) se duplicará también, mostrando un aumento falso en las distorsiones (entre utilidades y costos marginales), ya que ninguna variable macro o micro habrá cambiado. Para remediar esto busquemos una versión de (6) independiente del nivel de precios, basada en precios relativos y tasas de sustitución, en vez de precios absolutos utilidades marginales. Para ello, definamos las tasas marginales de sustitución con respecto a $x_{1}$ :

$$
\underline{\mathrm{s}} \equiv \underline{\mathrm{s}}(\underline{\mathrm{x}} ; h) \equiv u_{\underline{\mathrm{x}}} / u_{1}
$$

\footnotetext{
$p_{2} x_{2}$ como el costo de producción de aquello que se vende al público y $-p_{1} x_{1}$, o simplemente $-x_{1}$, como el precio total que se carga al consumidor por $x_{2}$.

El uso de $p$ como constantes solo se justifica al margen, y para encontrar su valor deb $\epsilon$ en general usarse una descripción global (no lineal) de la tecnología: véase Seade (1977).
} 
(donde $u_{1} \equiv u_{\mathrm{X}_{1}}$ ), cuyas derivadas parciales con respecto a $h$ (o sea el cambio de las pendientes de las curvas de indiferencia de distintas $h$ 's a través de un punto fijo $\underline{x}$ ) denotamos como $s_{h}$. Con esto y rearreglando (6) obtenemos

$$
\left.\underline{(\mathrm{s}}-\underline{\mathrm{p}} / p_{1}\right)=\frac{u_{1} \underline{\mathrm{s}}_{h}}{p_{1} f(h)} \mu(h)
$$

El lado izquierdo de esta expresión nos da la distorsión de precios que, en el óptimo, debe imponerse en las selecciones de un individuo $h$ entre cada bien $y$ el numerario. En efecto, $\left(\mathrm{s}-\mathrm{p} / p_{1}\right)$ es la diferencia entre los precios relativos que el Sr. $h$ enfrenta al margen (a los cuales iguala sus tasas marginales de sustitución) y los precios cargados (o sueldos pagados) por los productores), que el individuo percibiría en la ausencia de intervención fiscal. Es decir, $\left(\underline{\mathrm{s}}-\mathrm{p} / p_{1}\right)$ es precisamente el vector de impuestos marginales aplicados al consumo de los distintos bienes, en términos de numerario, en el óptimo.

Ciertas inferencias sobre el nivel de estos impuestos a distintos niveles de consumo pueden hacerse examinando (9), pero conviene dejar esto por el momento: la discusión de propiedades de los impuestos óptimos, en las secs. 4 y 5 , hace uso implícito de la estructura de (9).

La ecuación-vector (9) contiene una ecuación menos que (6), pues para el numerario, (9) es una identidad $0=0$. La ecuación restante en (6), aún "disponible", la usamos para encontrar $\mu$ en forma explícita. Para ello, nótese que el primer ecuación-elemento de (6),

$$
\mu^{\prime}+\mu \frac{u_{1 h}}{u_{1}}=\frac{\left(u_{1}-p_{1}\right) f}{u_{1}},
$$

es una ecuación diferencial lineal de primer orden, en $\mu$, que puede resolverse por los métodos usuales dando

$$
\mu(h)=\int_{\underline{h}}^{h}\left(1-\frac{p_{1}}{u_{1}}-\right) \exp \left(-\int_{h^{\prime}}^{h} \frac{u_{1 h}}{u_{1}} d h^{\prime \prime}\right) f\left(h^{\prime}\right) d h^{\prime},
$$

más un término en $\mu(\underline{h})$ (proveniente de la "constante de integración") que se hace cero, por (7).

\section{IMPUESTOS ÓPTIMOS: PROPIEDADES}

\section{Observaciones numéricas previas}

Las ecuaciones (3), (9) y (10) forman un sistema de $N+1$ ecuaciones diferenciales (e integrales) en las $N+1$ variables dependientes $\underline{x}(h)$ y $\mu(h)$. Este puede resolverse por métodos numéricos en casos particulares, es decir especificando la forma en que los consumidores difieren entre sí (el papel o significado de $h$ ), la forma funcional de u $(\mathrm{x} ; h)$ para cada uno de ellos y la distribución de $h$ en la 
población. Abundante investigación numérica sobre estas líneas se ha llevado a cabo, estudiando en especial el nivel general, más que la estructura, de la curva de impuestos, trabajando sobre los supuestos especiales del modelo original de Mirrlees. Dicho nivel tiende a aumentar, cual es de esperar, $a$ ) con el grado de desigualdad antes de impuestos [Mirrlees (1971)]; $b$ ) con la aversión a la desigualdad que el gobierno quiera imprimir a su política fiscal [Atkinson (1973)] y sobre todo c) con la inelasticidad de la oferta (compensada) de trabajo [Feldstein (1973) y Stern (1976)], que disminuye el efecto nocivo sobre los incentivos individuales que representa gravar el ingreso.

Sin embargo, una propiedad cualitativa de los impuestos óptimos, que se encontró sistemáticamente en estos estudios numéricos y que se trató de explicar a la luz de los supuestos específicos (funciones) adoptados, es su carácter regresivo en los rangos de ingresos altos, incluso cuando las preferencias fiscales que se adoptan son fuertemente redistributivas. Mi interés principal en lo que sigue es presentar y discutir un resultado analítico, no numérico, general, que explica este comportamiento.

\section{Resultados}

El resultado central es que, dados ciertos supuestos no restrictivos cuyo propósito es puramente técnico, ${ }^{8}$ en cada extremo de la función de impuestos en que no hay "agrupamiento" de $h$ 's diferentes en un mismo punto, el impuesto marginal (o cada impuesto marginal, si $N>2$ ) es cero. Como resultado, los impuestos óptimos no son, típicamente, progresivos (ni regresivos) a todo lo largo de la escala. Por "agrupamiento" ["bunching" en Seade (1977)] entendemos que toda $\mathrm{h}$ en un rango dado $\left[h_{0}, h_{1}\right]$ (con $\left.h_{1}>h_{0}\right)$ tiene los mismos consumos, como es el caso si por ejemplo hay desempleo en el arreglo óptimo, bajo el cual distintas gentes tendrían el mismo ingreso bruto (cero) y en consecuencia también el mismo ingreso neto (el seguro de desempleo que perciban $y / u$ otras transferencias pecuniarias o en especie). Dados (9) y (7), la prueba de este resultado consiste básicamente en probar que $\mu / f$ tiende a cero en los extremos, independientemente de si f tiende a cero o no o de qué tan rápido lo haga [detalles en Seade (1977)].

Este resultado puede verse como el caso extremo (y preciso) de una observación que, a grosso modo, puede hacerse en (9) y (10) sobre el nivel óptimo de impuestos en puntos interiores de la escala. En efecto, dichas ecuaciones nos dicen que la distorsión $\left(s_{i}-p_{i} / p_{1}\right)$ en un punto y para un bien dados, debe ser mayor a mayor $\partial s_{i} / \partial h$, a mayor $\mu(h)$ y a menor $f(h)$. El primero de estos factores obedece a razones claras, independientes del resto de la discusión, ${ }^{9}$ mientras que los otros

8 Básicamente, que diferencias pequeñas en $h$ se traducen en diferencias pequeñas en las preferencias es decir que $s_{h}$ es finita en la población, en el equilibrio óptimo) y que el dominio de la función de densidad $f(h)$ está acatado: que $\mathrm{f}($.$) no tiene "átomos" (proporciones$ notables de la población en un mismo valor de $h$ ), al menos en semivecindades de los extremos h, $h$.

$9 \partial s_{i} / \partial h$ es mayor, por definición, mientras más difiera la pendiente $s_{i}$ de individuos con $h$ 's similares (alrededor del x de equilibrio de una $h$ dada) lo cual, puede mostrarse, es equivalente a una baja sustitubilidad entre el bien en cuestión y el numerario. Esto, intuiti- 
dos factores, a primera vista menos transparentes, se prestan a una interpretación que reviste mayor interés y que dejamos para la sección siguiente. Baste notar por lo pronto que $\mu(h)$ será mayor, a grandes rasgos, mientras más difieran las valuaciones sociales (al margen) del consumo de distintos individuos (dispersión de $u_{1}$ ) y mientras mayor sea el número de gente $\int f\left(h^{\prime}\right)$ que hay hacia uno u otro extremo de la escala.

Conviene notar por lo pronto un segundo resultado, complemento natural del referente a los extremos de la curva de impuestos: que si se desea en principio llevar a cabo una redistribución en la dirección $\bar{h} \rightarrow \underline{h}$ (si la utilidad social marginal es decreciente en $h$ ), el impuesto marginal (o margen de ganancia, en el caso de una empresa pública) es estrictamente positivo, ${ }^{10}$ excepto en los extremos en que es cero. Esto se deduce mostrando que, de no ser así, (7) y (10) son incompatibles; por (7), $\left(1-p_{1} / u_{1}\right)$ debe cambiar signo al menos una vez (o ser idéntico a cero), y por el supuesto redistributivo esto debe traducirse en precisamente un cambio de signo, de $+\mathrm{a}-$. Por lo tanto, dado $\mu(\bar{h})=0$ en (7), la integral truncada en (10) (que pierde parte de los valores negativos de su integrando) debe ser siempre positiva.

De estos dos resultados se sigue que la forma típica de una curva de impuestos óptimos es en forma de $S$, con una tasa máxima interior que cae ${ }^{11}$ hacia cero en los extremos, como se ilustra en la figura 1.

\section{CONCLUSIONES PARA DISEÑO FISCAL}

\section{Interpretación}

La explicación de los resultados que propongo es la siguiente: todo impuesto marginal conlleva una ineficiencia, una pérdida de excedente del consumidor para el individuo afectado si se quiere, en el sentido de que ese individuo podría estar pagando el mismo impuesto total y obteniendo una canasta de consumo que él preferiría, bajo un sistema de precios con distorsión cero al margen (equivalentemente, podía estar obteniendo la misma "utilidad" y pagando más impuesto). La única razón por la que la distorsión se impone es para llevar a cabo una redistribución, de todos los consumidores arriba de ese punto, a todos los consumidores abajo ${ }^{12}$ (o, en principio, en la dirección opuesta, según sea deseable). Esto se ilustra en la figura 2: un aumento del impuesto total que se aplica a

vamente, permite aumentar la distorsión (y redistribución) fiscal sin aumentar el daño a incentivos, pues, los efectos sustitución (à la slutsky) son bajos, dominados por efectos-ingresos, siempre neutros.

$10 \mathrm{O}$, mejor, del mismo signo que $s_{h}$.

11 No necesariamente en forma monotónica, aunque las excepciones a esta regla (puntos de inflexión adicional en la fig. 1) parecerían extraños, propios por ejemplo de distribuciones de ingreso ex-ante bimodales.

12 Esto no cambia si el verdadero motivo es obtener ingreso para el erario, pues el mismo ingreso se podía haber obtenido, en principio, en una forma "no distorsionadora", cómo un tributo común a toda $h$, lo cual precisamente reemplazamos por uriá política de impuestos redistributiva como la descrita en el texto para lograr una menor depresión del consumo privado en los niveles bajos. 


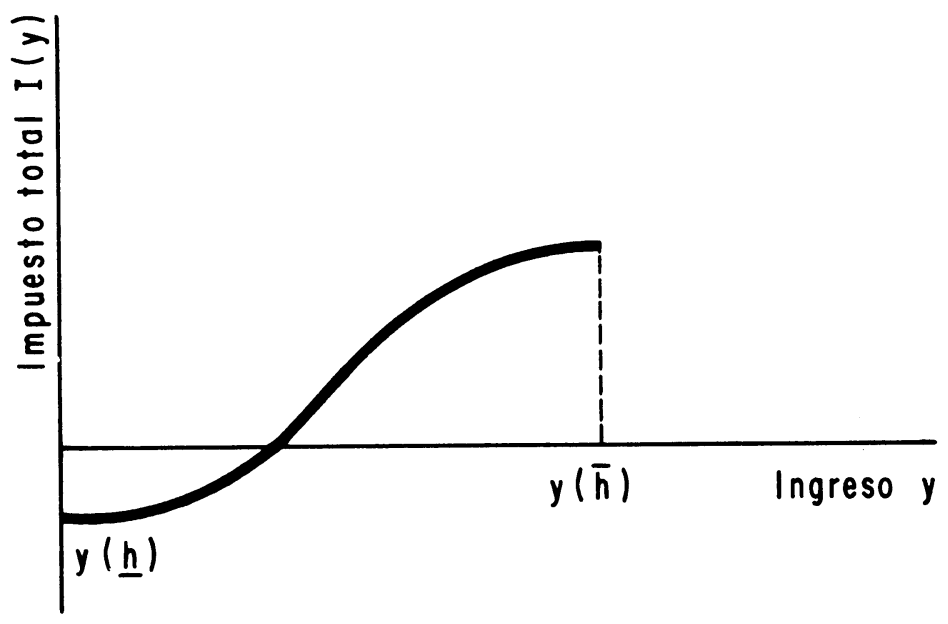

Figura 1. Curva 'típica' de impuestos óptimos (ilustrada para el caso de transferencia neta positiva al pobre, incluido en ella lo que en un esquema más detallado sería el gasto social del estado).

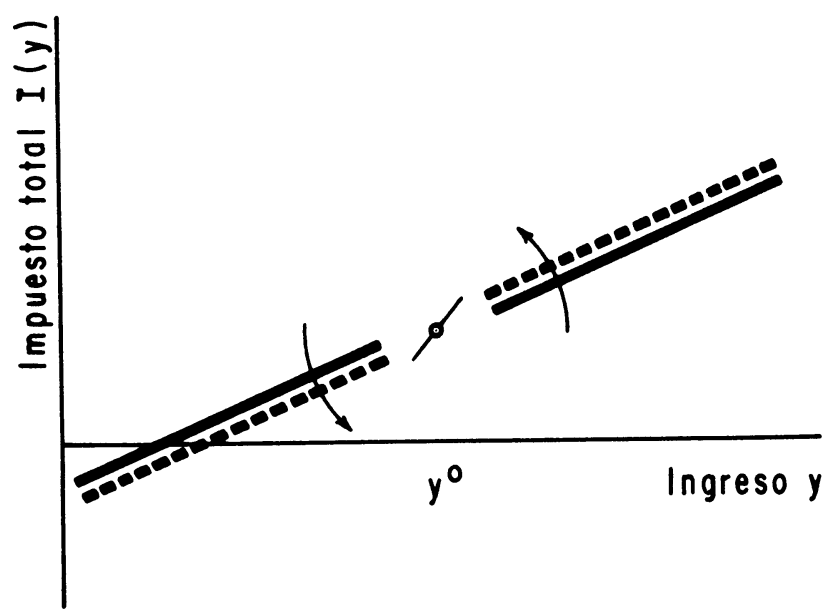

Figura 2. Efecto redistributivo 'de arriba a abajo' de un aumento en el impuesto marginal en ( $y$ alrededor de) $y^{\circ}$, con recaudación neta fija. 
ingresos $y>y^{\circ}$ (o sea un aumento del impuesto marginal en $y^{\circ}$ mismo) resulta, a balance fiscal constante, en una disminución del impuesto que el pobre paga o en un aumento del subsidio neto que recibe del Estado, sea que éste consista de una transferencia fiscal directa o se canalice a través de beneficios redistributivos incorporados en el gasto público.

Sin embargo, para que esta redistribución resultante de la distorsión en $\mathrm{y}^{\circ}$ tenga lugar, es necesario que los dos grupos entre los cuales se quiere redistribuir no estén vacíos: que haya gente arriba y abajo del punto $\mathrm{y}^{\circ}$ en cuestión. De otra manera sólo la pérdida de eficiencia prevalece y el impuesto es indeseable. Un impuesto en el extremo superior no afecta ingreso alguno y sólo reduce los incentivos al margen de individuos con ese nivel de ingresos y con ello su utilidad o bien la recaudación que de ellos podría obtenerse a utilidad constante. En forma similar, un impuesto al margen en el nivel de ingresos de los consumidores más pobres afecta a todos en la economía por igual y debe por tanto ser reemplazado por un impuesto general fijo, evitando así la pérdida de eficiencia (utilidad, o costo fiscal de esta utilidad) asociada con la distorsión.

Por otro lado la ganancia redistributiva que hemos argüido, desaparece en los extremos de la distribución, no desaparece en los puntos intermedios, por las mismas razones dadas. Esto explica el segundo resultado mencionado en la sección anterior, referente a la positividad de los impuestos marginales intermedios.

\section{Desempleo}

El argumento anterior desde luego no procede, en forma directa, si hay por ejemplo desempleo, aunque la explicación dada nos indica la forma en que el patrón (a diferencia de mero nivel) de impuestos directos debe relacionarse a esta variable macroeconómica. En efecto, con desempleo, un impuesto positivo "en el primer peso" de ingresos no afecta a toda la población como se dijo en el argumento anterior, sino sólo a todos aquellos cuyos ingresos brutos son positivos. Así, dicho impuesto estaría en beneficio del desempleado, de forma que el objetivo redistributivo no desaparece en el extremo inferior haciendo con ello deseable, puede demostrarse una distorsión base. Esta es una forma de "agrupamiento" de diferentes consumidores en un punto extremo (en este caso en $y=0$ ), excluido en el enunciado del resultado.

El interés del caso en el cual hay desempleo va sin embargo mas allá de la observación negativa anterior. Por una parte, puede mostrarse que el resultado (distorsión cero en los extremos) aún se obtiene en forma aproximada: distorsión baja si el desempleo también lo es, lo cual seguramente cubre los tipos no keynesianos de desempleo, tales como desempleo voluntario o friccionario. Pero esto no es verdadero desempleo. Por otra parte, si hay un nivel mayor de desempleo que no puede eliminarse a través de manejo macroeconómico en el mediano plazo, la imposición óptima se modifica en una forma interesante que tiene dos facetas:

a) El hecho mismo de que haya desempleo hace positivo al impuesto marginal base ${ }^{13}$ debido al argumento dado arriba: la recaudación adicional que se

13 Aproximadamente en proporción directa al nivel de desempleo. 
logra mediante un impuesto al ingreso base de todo individuo con empleo, juega un papel redistributivo importante en favor del desempleado. Si se quiere, el efecto sobre los incentivos a trabajar pierde fuerza cuando hay un excedente de mano de obra disponible. Más aún, puesto que el resto de la curva de impuestos óptimos no se ve afectada por el desempleo sino indirectamente, el efecto sobre el impuesto base se traduce en que el nivel de impuestos y de gasto público (transferencia base), y por tanto el nivel de intervención estatal (en el sector consumo) deben también aumentar con, y en proporción al nivel del desempleo.

b) Ahora bien, una vez rechazado el impuesto cero, a causa del desempleo, se hace necesario determinar lo que lo ha de sustituir. Básicamente, ${ }^{14}$ la respuesta es tan simple que el resultado original: el impuesto base debe ser mayor y mayor (con las ganancias distributivas que esto ofrece) en tanto subsista el desempleo involuntario, es decir hasta alcanzar el punto en que el número de gentes que optan por trabajar (dados los impuestos que enfrentan y el consumo garantizado al desempleado) sea igual al empleo disponible. Esto se debe, intuitivamente, a que en tanto todo aquél con empleo prefiera estrictamente su situación a perder su empleo, será posible gravarle un poco más ese excedente del consumidor de que goza, para transferirlo (como consumo adicional) al desempleado. La prueba es simple pero basada en un diagrama un tanto laborioso (similar a la fig. 2 en Seade (1977)) que será mejor omitir.

\section{De los extremos al interior de la escala: inferencias sobre la forma ótima de gravar el ingreso}

Finalmente, dejando de lado consideraciones de desempleo que pueden incorporarse en una versión modificada de la discusión que sigue, es importante determinar qué tan local es el resultado sobre los puntos extremos, y más en general, qué es lo que este resultado y su interpretación nos dicen sobre el patrón óptimo de progresividad.

Nuestra interpretación del resultado para los puntos extremos, en términos de pérdida de eficiencia local contra ganancia redistributiva global, indica que el impuesto marginal a dos o tres deciles del extremo inferior de la escala de ingresos, por ejemplo, debe ser menor desde el punto de vista de consideraciones de eficiencia mientras mayor sea la densidad (la multiplicidad de la distorsión) en ese punto o rango de ingresos y que debe ser también menor, desde el punto de vista de consideraciones distributivas, mientras menor sea la dispersión de consumidores de ese punto hacia abajo, pues en ese caso las ganancias por redistribución dentro de ese rango son mínimas - unos y otros son igualmente pobres y los beneficios o transferencias para todos ellos deben ser financiados principalmente a través de impuestos en ingresos más altos. Ahora bien, ambas características de la forma de la distribución $f(h)$ que acabamos de mencionar tienden a darse juntas aunque $f^{\prime} s$ con muchas curvas son en principio posibles), como resultado del habitual sesgo a la derecha de distribuciones de ingreso observadas. Por

14 Estamos suponiendo, para evitar complicaciones de poco interés, que en el óptimo (con control sobre el nivel de desempleo) no hay desempleo. Esto requiere una aversión limitada al trabajo por parte del trabajador de ingresos bajos; véase la sec. 3 de Seade (1977). 
tanto es de esperar que, en el extremo inferior de la escala de ingresos, el impuesto marginal óptimo en un rango amplio de ingresos sea bajo. No se puede ser más categórico que esto sin antes adoptar formas específicas de $u$ (.) y $f($.), pero ésta es una clara conclusión cualitativa que se aplica a funciones de distribución comunes, tales como la Normal Logarítmica. De acuerdo con esto y muy en contra de la propuesta usual, el impuesto negativo al ingreso (negative income tax) debería consistir esencialmente de una transferencia fija general, sin acompañar a ésta de un aumento en el impuesto marginal que los trabajadores de ingresos bajos o medios-bajos enfrentan - aunque claro está que el balance total del erario exigiría disminuir el nivel de exención y hacer otros ajustes "globales". La base principal de recaudación directa deben ser, como en la figura 1 , los ingresos medios y medios-altos.

Por otro lado, es de suponer que la propiedad "distorsión-cero" del extremo superior sea una aproximación bastante pobre de la forma en que los ingresos altos, en general, deben gravarse. En cada punto de la cola superior (excepto muy cerca del punto extremo, que puede incluso no conocerse sino grosso modo), típicamente larga y delgada, hay muy poca gente sufriendo el esfecto eficiencia de un impuesto marginal positivo, en tanto que la recaudación derivada de éste será considerable, dada la alta dispersión a la derecha.

\section{Bibliografía}

Atkinson, A. B. (1973), "How Progressive should Income-Tax be?", en M. Parkin, editor, Fssays in Modern Economics (Longman, Londres), pp. 90-109.

Feldstein, M. S. (1973), "On the Optimal Progressivity of the Income Tax", Journal of Public Economics, vol. 2, pp. 357-376

MirrleEs, J. A. (1971), "An Exploration in the Theory of Optimum Income Taxation", Review of Economic Studies, vol. 38, pp. 175-208.

Pigou, A. C. (1929), The Economics of Welfare; referencias a la cuarta edición (1946; MacMillan, Londres).

SeAdE, J. (1977), "On the Shape of Optimal Tax Schedules", Journal of Public Economics, vol. 7 pp. 203-235.

SEAdE, J. (1980), "Optimal Nonlinear Policies for Non-Utilitarian Motives", en D. Collard et. al., comps., The Limits to Redistribution (Colston Research Society 1979: Scientechnica, Bristol).

SEN, A. K. (1973), On Economic Inequality (Warwick Radcliffe Lectures 1972: Oxford University Press, Oxford).

STERn, N. H. (1976), "On the Specification of Models of Optimum Income Taxation", Journal of Public Economics, vol. 6, pp. 123-162. 